\title{
A MATRIX INVERSE
}

\author{
D. M. BRESSOUD ${ }^{1}$
}

\begin{abstract}
ABSTRACr. George Andrews has demonstrated that the Bailey transform is equivalent to the inversion of an infinite-dimensional matrix whose entires are rational functions in $q$. We generalize this inversion by introducing an extra parameter which brings much greater symmetry.
\end{abstract}

Let $A=\left\{A_{n k}\right\}_{n, k=0}^{\infty}$ be an infinite-dimensional lower triangular matrix; $k>n$ implies that $A_{n k}=0$. We say that $A$ has an inverse, written $A^{-1}=\left\{A_{n k}^{-1}\right\}$, if

$$
\sum_{k=m}^{n} A_{n k} A_{k m}^{-1}=\delta_{n m},
$$

for all nonnegative $n$ and $m, m \leqslant n$. The inversion of such matrices when the entries are rational functions in $q$ plays an important role in $q$-series identities.

Andrews [1] has shown that the Bailey transform [2,3] used to prove and generalize the Rogers-Ramanujan identities is equivalent to the following matrix inversion.

Let $B=\left\{B_{n k}\right\}_{n, k=0}^{\infty}$ where

$$
B_{n k}=\frac{1}{(q)_{n-k}(a q)_{n+k}},
$$

$(a)_{\infty}=\prod_{i=0}^{\infty}\left(1-a q^{i}\right),(a)_{m}=(a)_{\infty} /\left(a q^{m}\right)_{\infty}$. Then $B^{-1}=\left\{B_{n k}^{-1}\right\}$ where

$$
B_{n k}^{-1}=\frac{\left(1-a q^{2 n}\right)(a)_{k+n}(-1)^{n-k} q^{(n-k)}}{(1-a)(q)_{n-k}} .
$$

More recently, Gessel and Stanton proved a number of $q$-series identities using this same inversion (Theorem 1.2 of [5]).

Andrews' inversion is a special case of a far more appealing result.

THEOREM. Let $D=\left\{D_{n k}(a, b)\right\}_{n, k=0}^{\infty}$ where

$$
D_{n k}(a, b)=\frac{\left(1-a q^{2 k}\right)(b)_{k+n}\left(b a^{-1}\right)_{n-k}\left(b a^{-1}\right)^{k}}{(1-a)(a q)_{k+n}(q)_{n-k}} .
$$

Then $D^{-1}=\left\{D_{n k}(b, a)\right\}_{n, k=0}^{\infty}$.

Received by the editors October 28, 1982.

1980 Mathematics Subject Classification. Primary 33A30; Secondary 05A17, 10A45, 05 A15.

'Partially supported by National Science Foundation and Sloan Foundation. Research done at University of Wisconsin, Madison. 
For each $n=k \geqslant 0$, if the $k$ th column of $D(a, b)$ is divided by

$$
\frac{1-a q^{2 k}}{1-a}\left(b a^{-1}\right)^{k}
$$

and the $n$th row of $D(b, a)$ is multiplied by

$$
\frac{1-a q^{2 n}}{1-a}\left(b a^{-1}\right)^{n}
$$

then we get two matrices which are inverses to each other and which give Andrews' inversion when $b=0$. The inversion in this theorem is equivalent to the transform given by the author in [4].

Proof of Theorem. We compute the inner product of the $n$th row of $D$ with the $m$ th column of $D^{-1}$. If $m>n$, then the inner product is trivially zero and so we assume that $m \leqslant n$. (a)

$$
\begin{aligned}
& \sum_{k=m}^{n} D_{n k}(a, b) D_{k m}(b, a) \\
& =\sum_{k=m}^{n} \frac{\left(1-a q^{2 k}\right)(b)_{k+n}\left(b a^{-1}\right)_{n-k}\left(b a^{-1}\right)^{k}\left(1-b q^{2 m}\right)(a)_{m+k}\left(a b^{-1}\right)_{k-m}\left(a b^{-1}\right)^{m}}{(1-a)(a q)_{k+n}(q)_{n-k}(1-b)(b q)_{m+k}(q)_{k-m}}
\end{aligned}
$$

Setting $k=j+m$ and pulling out of the summation those factors which do not depend on $j$ yields

$$
\begin{aligned}
\sum D_{n k}(a, b) D_{k m}(b, a)=\frac{\left(1-a q^{2 m}\right)\left(1-b q^{2 m}\right)(b)_{m+n}\left(b a^{-1}\right)_{n-m}(a)_{2 m}}{(1-a)(1-b)(a q)_{m+n}(q)_{n-m}(b q)_{2 m}} \\
\cdot \sum_{j=0}^{n-m} \frac{\left(1-a q^{2 m+2 j}\right)\left(b q^{m+n}\right)_{j}\left(a q^{2 m}\right)_{j}\left(a b^{-1}\right)_{j}\left(q^{n-m-j+1}\right)_{j}\left(b a^{-1}\right)^{j}}{\left(1-a q^{2 m}\right)\left(b a^{-1} q^{n-m-j}\right)_{j}\left(a q^{n+m+1}\right)_{j}\left(b q^{2 m+1}\right)_{j}(q)_{j}}
\end{aligned}
$$

If $m=n$, the summation reduces to a single term equal to one and the entire right side of (2) is easily seen to equal one. If $m<n$, it is sufficient to show that the summation on the right of (2) is zero. By the limiting form of Jackson's theorem (equation $A 5$ in [4]), this summation is equal to

$$
\frac{\left(a q^{2 m+1}\right)_{n-m}\left(q^{m-n+1}\right)_{n-m}}{\left(a b^{-1} q^{m-n+1}\right)_{n-m}\left(b q^{2 m+1}\right)_{n-m}}
$$

which is zero since $m<n$. 


\section{REFERENCES}

1. G. E. Andrews, Connection coefficient problems and partitions, Proc. Sympos. Pure Math., vol. 34, Amer. Math. Soc., Providence, R. I., 1979, pp. 1-24.

2. W. N. Bailey, Some identities in combinatory analysis, Proc. London Math. Soc. (2) 49 (1947), 421-435.

3. Identities of the Rogers-Ramanujan type, Proc. London Math. Soc. (2) 50 (1949), 1- 10.

4. D. M. Bressoud, Some identities for terminating q-series, Math. Proc. Cambridge Philos. Soc. 89 (1981), 211-223.

5. I. Gessel and D. Stanton, Applications of $q$-Lagrange inversion to basic hypergeometric series, Trans. Amer. Math. Soc. (to appear).

Department of Mathematics, Pennsylvania State University, University Park, Pennsylvania 16802 